\title{
Re-examining the Gamma-ray Properties of Globular Clusters
}

\author{
Kwangmin $\mathrm{Oh}^{* \dagger}$ \\ The Department of Astronomy, Space Science and Geology, Chungnam National University, \\ Daejeon 34134, South Korea \\ E-mail: min8582046@gmail.com \\ C. Y. Hui ${ }^{\ddagger}$ \\ The Department of Space Science and Astronomy, Chungnam National University, Daejeon \\ 34134, South Korea \\ E-mail: cyhui@cnu.ac.kr
}

We have re-examined the properties of $\gamma$-ray emitting globular clusters (GCs) with the data obtained by Fermi Large Area Telescope in the past nine years. With an updated sample of 19 $\gamma$-ray GCs, we have performed a statistical analysis in the framework set by Hui et al. (2011) [1]. We confirm that the correlation between the $\gamma$-ray luminosities $L_{\gamma}$ and the metalicity $[\mathrm{Fe} / \mathrm{H}]$ is as significant as that between $L_{\gamma}$ and the two-body encounter frequencies $\Gamma_{c}$. Correlations between $L_{\gamma}$ and the energy densities of the soft photon field $u_{\text {optical }} / u_{\mathrm{IR}}$ at location of the GCs have also been confirmed. With a two-dimensional regression analysis, we have revised the fundamental planes of $\gamma$-ray GCs which relate $L_{\gamma},[\mathrm{Fe} / \mathrm{H}] / \Gamma_{c}$ and $u_{\text {optical }} / u_{\mathrm{IR}}$. The updated relations based on can provide better prediction of the $\gamma$-ray properties of GCs and support the scenario that the $\gamma$-ray emission is originated from the inverse Compton scattering (ICS) between the pulsar wind and the ambient soft photon fields [2].

7th Fermi Symposium 2017

15-20 October 2017

Garmisch-Partenkirchen, Germany

\footnotetext{
*Speaker.

${ }^{\dagger}$ Oh Kwangmin is supported by BK21 PLUS Chungnam National University.

${ }^{\ddagger}$ CYH is supported by the National Research Foundation of Korea grant 2016R1A5A1013277
} 


\section{Introduction}

Globular clusters (GCs) are efficient factories for producing millisecond pulsars (MSPs) through dynamical formation processes [3]. As MSPs have been established as a new class of $\gamma$-ray emitters, it is not surprise that GCs also emit $\gamma$-ray emission [5]. The $\gamma$-ray properties of GCs have been examined in previous works (e.g. [1], [4]). Apart from the positive correlations for $L_{\gamma}-\Gamma_{c}$ and $L_{\gamma}-[\mathrm{Fe} / \mathrm{H}]$ which are expected from scaling with the MSP population in a GC [3], correlations have also been found between $L_{\gamma}$ and the energy densities of the soft photon field at location of the GCs. This supports the scenario that the $\gamma$-rays from GCs are originated from the inverse Compton scattering (ICS) between the pulsar wind and the ambient soft photon fields [2].

In the light of enlarged sample resulted from the uninterrupted observation with Fermi Large Area Telescope (LAT), it is timely to perform a statistical analysis to cross-check the results reported by [1] and, [4]. The results are reported in this paper.

\section{Updated Sample}

$19 \gamma$-ray GCs are adopted in our sample. They are collected from the 3FGL catalog [5] and Zhang et al. (2016) [6]. Their physical properties are summarized in Table 1. We have excluded NGC6624 and M28 from our sample as their $\gamma$-rays are dominated by a single MSP in the cluster (cf. [5] and the references therein). We have also excluded 2MS-GC01 in our analysis as there is no estimate of $[\mathrm{Fe} / \mathrm{H}]$ for this $\mathrm{GC}$.

For those $\gamma$-ray GCs reported in Tam et al. (2011) [7] but are excluded in the lists in [5] and [6] (e.g. Liller 1), we have performed searches for their $\gamma$-ray emission with nine years Fermi LAT data. However, we do not found any significant signal from these GCs and therefore they are also excluded in this work. Hence, our adopted sample is different from [6] which include all the GCs reported by [7] in their statistical analysis.

\section{Statistical Analysis}

In Figure 1, we show the relations between $L_{\gamma}$ and four cluster parameters. We have also performed a non-parametric correlation analysis and the Spearman rank coefficients $\rho$ and the corresponding $p$-values are given in each panel. We can see that both $[\mathrm{Fe} / \mathrm{H}]$ and $\Gamma_{c}$ are strongly correlated with $L_{\gamma}$. Although the significance is weaker, evidence for the correlations between $L_{\gamma}$ and the ambient soft photon energy densities as suggested by [1] and [4] has also been confirmed.

We proceeded to perform the 2-D regression to obtained the updated fundamental plane relations, which are given as follows:

$$
\begin{aligned}
& \log L_{\gamma}=(34.39 \pm 0.12)+(0.55 \pm 0.14) \log \Gamma_{c}+(0.55 \pm 0.22) \log u_{\text {optical }} \\
& \log L_{\gamma}=(34.78 \pm 0.16)+(0.58 \pm 0.15) \log \Gamma_{c}+(0.57 \pm 0.33) \log u_{\mathrm{IR}} \\
& \log L_{\gamma}=(35.39 \pm 0.32)+(0.60 \pm 0.20)[\mathrm{Fe} / \mathrm{H}]+(0.17 \pm 0.29) \log u_{\text {optical }} \\
& \log L_{\gamma}=(35.52 \pm 0.22)+(0.68 \pm 0.19)[\mathrm{Fe} / \mathrm{H}]+(-0.07 \pm 0.40) \log u_{\mathrm{IR}}
\end{aligned}
$$

The edge-on views of these relations are given in Figure 2. 
Table 1: Properties of the Gamma-ray emitting GCs

\begin{tabular}{ccccccc}
\hline Cluster Name & $\begin{array}{c}d^{(\mathbf{a})} \\
(\mathrm{kpc})\end{array}$ & {$[\mathrm{Fe} / \mathrm{H}]^{(\mathbf{a})}$} & $\log \Gamma^{(\mathbf{a})}$ & $\begin{array}{c}\log u_{\mathrm{IR}}(\mathbf{b}) \\
\left(\mathrm{eV} \mathrm{cm}^{-3}\right)\end{array}$ & $\begin{array}{c}\log u_{\text {optical }}(\mathbf{b}) \\
\left(\mathrm{eV} \mathrm{cm}^{-3}\right)\end{array}$ & $\begin{array}{c}\log L_{\gamma}^{(\mathbf{c})} \\
\left(\mathrm{erg} \mathrm{s}^{-1}\right)\end{array}$ \\
\hline 47 Tuc & 4.5 & -0.72 & 0.67 & 0.22 & 0.81 & $34.68_{-0.13}^{+0.12}$ \\
Omega Cen & 5.2 & -1.53 & -0.17 & 0.41 & 1.30 & $34.44_{-0.15}^{+0.13}$ \\
M80 & 10.0 & -1.75 & 0.46 & 0.32 & 1.72 & $34.92_{-0.51}^{+0.28}$ \\
M62 & 6.8 & -1.18 & 1.02 & 0.77 & 6.55 & $35.04_{-0.14}^{+0.12}$ \\
NGC 6388 & 9.9 & -0.55 & 1.13 & 0.66 & 3.78 & $35.41_{-0.25}^{+0.12}$ \\
Terzan 5 & 6.9 & -0.23 & 0.72 & 1.28 & 11.1 & $35.41_{-0.19}^{+0.17}$ \\
NGC 6440 & 8.5 & -0.36 & 0.94 & 0.92 & 8.96 & $35.38_{-0.19}^{+0.15}$ \\
NGC 6441 & 11.6 & -0.46 & 1.17 & 0.73 & 3.25 & $35.57_{-0.12}^{+0.12}$ \\
NGC 6541 & 7.5 & -1.81 & 0.16 & 0.58 & 4.23 & $34.54_{-0.33}^{+0.24}$ \\
NGC 6652 & 10.0 & -0.81 & -0.35 & 0.48 & 3.03 & $34.89_{-0.15}^{+0.18}$ \\
NGC 6752 & 4.0 & -1.54 & 0.15 & 0.46 & 1.75 & $34.14_{-0.30}^{+0.19}$ \\
M15 & 10.4 & -2.37 & 0.83 & 0.1 & 0.38 & $34.73_{-0.10}^{+0.11}$ \\
NGC 6397 & 2.3 & -2.02 & -0.31 & 0.57 & 1.48 & $33.30_{-0.26}^{+0.33}$ \\
M5 & 7.5 & -1.29 & -0.22 & 0.17 & 0.77 & $34.34_{-0.08}^{+0.08}$ \\
M12 & 4.8 & -1.37 & -1.07 & 0.41 & 1.71 & $33.98_{-0.15}^{+0.09}$ \\
NGC 6139 & 10.1 & -1.65 & 0.29 & 0.65 & 3.42 & $34.71_{-0.19}^{+0.19}$ \\
NGC 2808 & 9.6 & -1.14 & 0.68 & -0.92 & -0.42 & $34.72_{-0.10}^{+0.08}$ \\
NGC 6316 & 10.4 & -0.45 & -0.23 & -0.14 & 0.68 & $35.41_{-0.06}^{+0.05}$ \\
NGC 6717 & 7.1 & -1.26 & -0.69 & -0.20 & 0.64 & $34.36_{-0.15}^{+0.11}$ \\
\hline
\end{tabular}

Note:(a) GC properties collected from Harris (1996, 2010 revision) [8]. The two-body enounter rate is estimated as $\Gamma_{c} \equiv \rho_{c}^{1.5} r_{c}^{2}$ where $\rho_{c}$ is the central luminosity density and $r_{c}$ is the core radius.(b) The soft photon densities are estimated by using GALPROP code[9]. (c) $L_{\gamma}$ in $0.1-300 \mathrm{GeV}$ [5][6].

\section{Conclusion}

With the updated and enlarged population of $\gamma$-ray GCs, we have re-examined the relations of $L_{\gamma}$ with a number of physical parameters. Our results support the assertion which have been previously made by [1], [2] and [4]: The $\gamma$-rays from a GC is a result of ICS between the relativistic wind from its MSPs, which are formed dynamically, and its ambient soft photon field.

\section{References}

[1] C.Y. Hui, et al. The Fundamental Plane of Gamma-ray Globular Clusters ApJ, 726, 100 (2011) [arXiv:1101.4107].

[2] K.S. Cheng, et al. The Origin of Gamma Rays from Globular Clusters ApJ, 723, 1219 (2010) [arXiv: 1009.2278].

[3] C.Y. Hui, et al. Dynamical Formation of Millisecond Pulsars in Globular Clusters ApJ, 714, 1149 (2010) [arXiv:1003.4332].

[4] P.H.T. Tam, et al. Gamma-ray Emission from Globular Clusters JASS, 33, 1 (2016) [arXiv:1207.7267].

[5] F. Acero, et al. Fermi large area telescope third source catalog ApJS, 218, 23 (2015) [arXiv:1501.02003].

[6] Zhang P, et al. Detection of Gamma-ray Emission from Globular Clusters M15, NGC 6397, 5904, 6218 and 6139 with Fermi-LAT MNRAS, 459, 99 (2016) [arXiv:1603.02358].

[7] P.H.T. Tam, et al. Gamma-ray Emission from the Globular Clusters Liller 1, M80, NGC6139, NGC 6541, NGC 6624, and NGC 6752 ApJ, 729, 90 (2011) [arXiv:1101.4106]. 

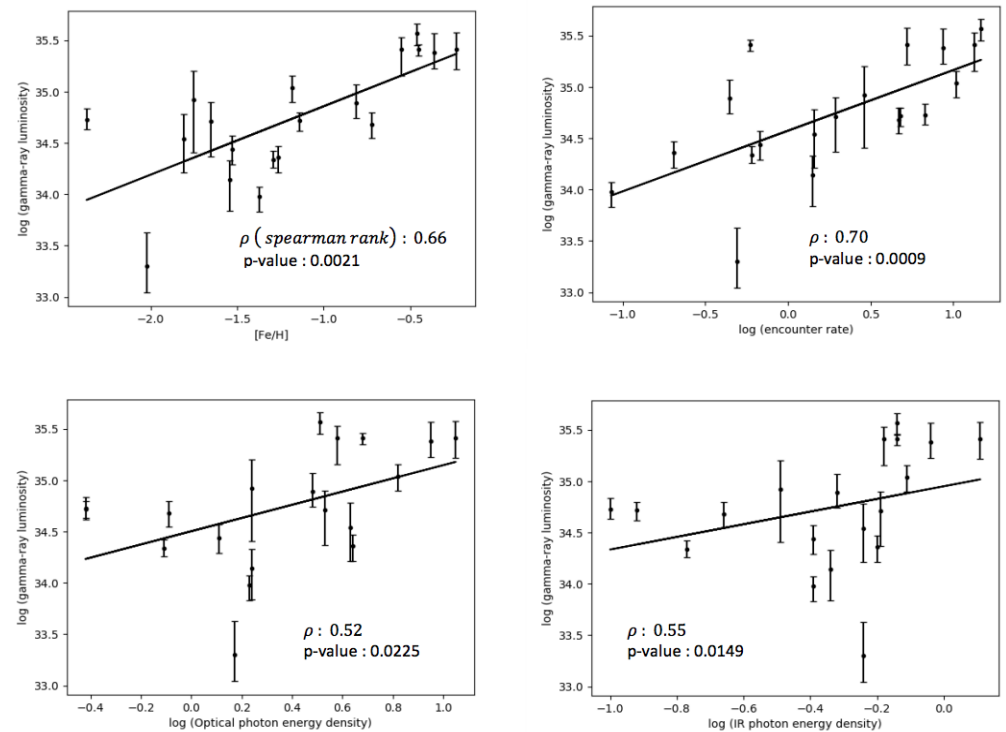

Figure 1: $\gamma$-ray luminosity vs individual GCs properties. Straight lines represent best-fit lines from the linear regression. The Spearman rank coefficients and the corresponding $p$-values are given in each panel.
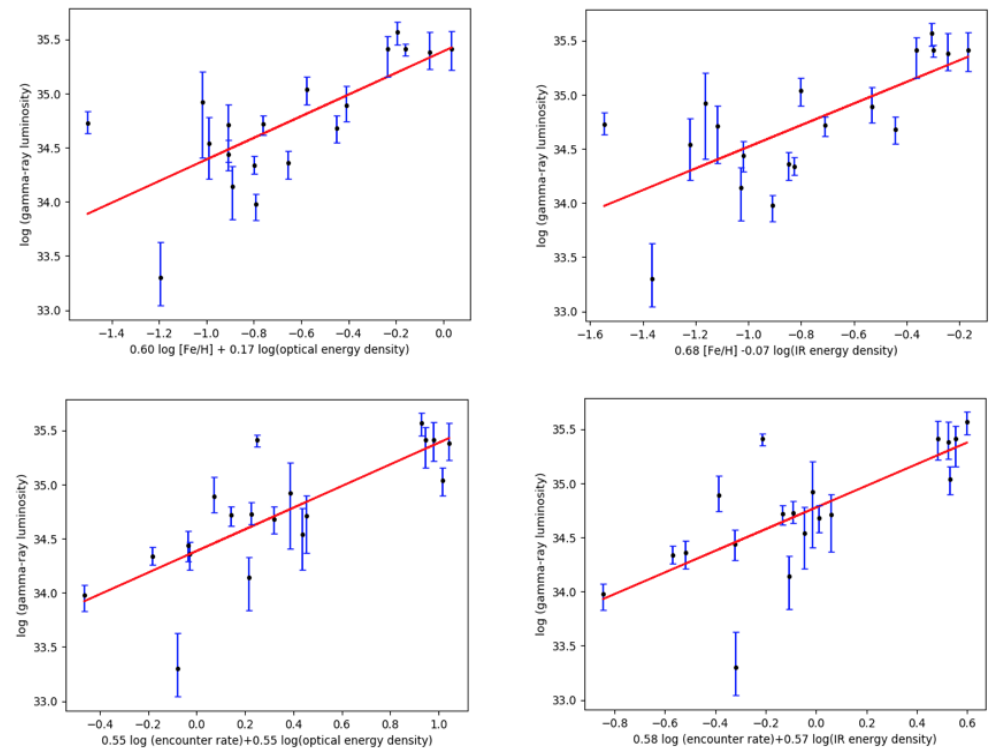

Figure 2: Edge-on views of the fundamental-plane relations of $\gamma$-ray GCs based on the updated and expanded sample.

[8] W.E. Harris, A Catalog of Parameters for Globular Clusters in the Milky Way AJ, 112, 1487 (1996)

[9] Strong AW, \& Moskalenko IV, Propagation of Cosmic-Ray Nucleons in the Galaxy ApJS, 509, 212 (1998)[arXiv:astro-ph/9807150]. 